\title{
Comparative Study between Subfoveal Choroidal Thickness in Glaucomatous Eyes Versus Normal Eyes Using Spectral Domain Optical Coherence Tomography
}

\author{
SAFAA GALAL, M.Sc.; MOHAMMAD M. KHAFAGY, M.D.; HEBA M. AHMED, M.D. and \\ SOHEIR M. ESMAT, M.D.
}

The Department of Ophthalmology, Faculty of Medicine, Cairo University

\begin{abstract}
Background: There has been increasing interest in investigating the role of the choroid in the pathogenesis of glaucomatous optic neuropathy. While measurement of choroidal thickness by spectral domain optical coherence tomography (SD-OCT) would be an attractive addition to the armamentarium of diagnostic and monitoring tools in glaucoma, the potential role of choroidal thickness as a risk factor requires further investigation.
\end{abstract}

Aim of the Study: To compare subfoveal choroidal thickness measurements in primary angle closure glaucoma (PACG), primary open angle glaucoma (POAG) patients, and normal control group, and to correlate between the subfoveal choroidal thickness with each of the average peripapillary retinal nerve fiber layer (RNFL) thickness, the average ganglion cell complex (GCC) thickness, as well as Humphrey field Analyzer parameters in these patients mainly the mean deviation (MD) and pattern standard deviation (PSD).

Patients and Methods: Cross sectional observational casecontrol study. The study included 124 eyes; 40 eyes of PACG patients, 42 eyes of POAG patients and 42 eyes of normal controls. Measurement of the subfoveal choroidal thickness was done using SD-OCT in The Investigative and Laser Unit of The Ophthalmology Department of Kasr Al-Ainy Hospital.

Results: There was no significant difference in subfoveal choroidal thickness among normal eyes, eyes with POAG and eyes with PACG (All $p$-values $>0.05$ ). There was a significant positive correlation between choroidal thickness and average RNFL thickness ( $r=0.804, p$-value $<0.001)$.

Conclusion: Lack of a significant difference in subfoveal choroidal thickness among normal eyes, eyes with POAG and eyes with PACG. A significant positive correlation existed between choroidal thickness and average RNFL thickness.

Key Words: Subfoveal choroidal thickness - Glaucoma Spectral domain optical coherence tomography.

\section{Introduction}

GLAUCOMA is a neurodegenerative optic neuropathy caused by injury to retinal ganglion cells

Correspondence to: Dr. Safaa Galal, The Department of Ophthalmology, Faculty of Medicine, Cairo University and their axons [1]. This cellular damage causes progressive changes to the optic disc, the retinal nerve fiber layer, the ganglion cell-inner plexiform layer, and the peripheral visual field, followed by the central field [1]

Elevated intraocular pressure (IOP) is the major risk factor for glaucoma, and its reduction can slow the progression of vision loss even in normal tension glaucoma (NTG) [2]. On the other hand, loss of visual function may continue even with medically or surgically controlled IOP indicating that IOP-independent factors such as worsened choroidal blood flow parameters may play a role in ocular hypertension, NTG, or primary open angle glaucoma (POAG) $[3,4]$. Other studies have reported that patients experiencing glaucoma progression have worse ocular hemodynamics than nonprogressors [5].

There has been increasing interest in investigating the role of the choroid in the pathogenesis of glaucomatous optic neuropathy (GON). Previous studies have found an association between GON and impaired choroidal circulation or blood flow to the optic nerve head $(\mathrm{ONH})$ [5].

Both the macular choroidal thickness and the peripapillary choroidal thickness measured by enhanced depth imaging optical coherence tomography (EDI-OCT) were correlated with inconsistent results in both primary open angle and angle closure glaucoma [6-8]. While Mwanza et et al. [6] did not find an association between subfoveal choroidal thickness and glaucoma in their group of patients, Bron et al., [7] reported thinner subfoveal choroidal thickness in glaucoma patients than normal controls. In addition, it has been suggested that patients with normal tension glaucoma had significantly thinner peripapillary choroidal thickness compared 
to normal [8]. Choroidal imaging by the EDI-OCT technique may serve as a tool to investigate the vascular theory of glaucoma pathogenesis.

Adequate visualization of the choroid using spectral domain optical coherence tomography (SD-OCT) has not been possible until recently, due to its posterior location and the presence of pigmented cells that attenuate the incident light. In 2008, Spaide et al., [9] were the first to visualize the choroid by inverting SD-OCT images, SDOCT images are highest in resolution near the zero-delay line. Thus, when the image is inverted, even if some resolution is lost at the vitreous and the retina, the resolution at the choroid is excellent [9]

Branchini, et al., [10] investigated the reproducibility of choroidal thickness measurements in normal subjects on three Spectral Domain Optical Coherence Tomography (SD-OCT) instruments, Heidelberg Spectralis, Zeiss Cirrus HD-OCT (Carl Zeiss Meditec Inc, Dublin, CA, USA), and OptovueRTVue (Optovue Inc., Fremont, CA, USA). They found that in their population of young healthy adults with normal vision, there was good reproducibility between choroidal thickness measurements of images acquired with Cirrus, Spectralis and RTVue. In addition, there was good interobserver correlation for all choroidal thickness measurements [10].

\section{Patients and Methods}

This cross sectional observational case-control study was conducted in The Investigative and Laser Unit of The Ophthalmology Department of Kasr Al-Ainy Hospital during the year 2016.

Our study included 124 eyes. Participants were divided into three groups:

- Group A: Included 40 eyes of chronic recurrent primary angle closure glaucoma (PACG) patients.

- Group B: Included 42 eyes of primary open angle glaucoma (POAG) patients.

- Group C: Included 42 eyes of normal controls.

Inclusion criteria:

- Age $\geq 40$ years.

- For group A: PACG patients: Eyes with:

- Narrow angle (defined as eyes in which the posterior trabecular meshwork was not seen for at least $180^{\circ}$ on indentation gonioscopy in the primary position).
- PAS and/or raised IOP (>21 mm Hg).

- Glaucomatous optic neuropathy (defined as a vertical cup/disc (C/D) ratio >0.7 and/or C/D asymmetry $>0.2$ and/or focal notching).

- Visual field changes on static automatedperimetry in the form of:

- Glaucoma hemifield test outside normal limits,

- Abnormal pattern and a SD of $p$-value less than $5 \%$ in the healthy population and

- Fulfilling test reliability criteria (fixation losses $<20 \%$, false positives $<33 \%$, and/or false negatives $\leq 33 \%$ ).

- History of previous acute attack(s) of angle closure glaucoma.

- For group B: POAG patients: Eyes with:

- Open angle (> grade 2).

- Raised IOP (>21 mm Hg).

- Glaucomatous optic neuropathy (defined as a vertical cup/disc (C/D) ratio > 0.7 and/or C/D asymmetry $>0.2$ and/or focal notching).

- Visual field changes on static automatedperimetry in the form of:

- Glaucoma hemifield test outside normal limits.

- Abnormal pattern and a SD of $p$-value less than $5 \%$ in the healthy population.

- Fulfilling test reliability criteria (fixation losses $<20 \%$, false positives $<33 \%$, and/or false negatives $\leq 33 \%$ ).

- For group C: Normal controls:

- No prior history of eye disease other than cataract not affecting the OCT image quality.

- No intraocular surgery.

- No glaucomatous optic neuropathy.

- IOP not exceeding $21 \mathrm{~mm} \mathrm{Hg}$.

\section{Exclusion criteria:}

- Any media opacity affecting the OCT image quality.

- Retinal diseases including diabetic retinopathy, age-related macular degeneration, or retinal vein occlusion.

- High myopia (>6 D).

- History of ocular trauma.

- History of ocular inflammation.

- History of ocular surgery (Except glaucoma surgery in PACG patients).

- IOP level $\leq 12 \mathrm{mmHg}$ (to exclude hypotony effect). 
All participants received a thorough explanation of the study design and aims. Study participants gave informed consent before initiation of any study-related procedures, and the study was conducted in compliance with informed consent regulations.

All patients were subjected to a full ophthalmological examination, including full medical and ophthalmic history taking, assessment of best corrected visual acuity (BCVA), external examination, slit lamp examination, assessment of the intraocular pressure using Goldmann applanation tonometry, and dilated fundus examination using slit lamp biomiocroscopy with a+90 D lens and the indirect ophthalmoscope with a+20 D lens.

The Humphery field analysis was done with the Swedish Interactive Thresholding algorithm (SITA) standard 24-2 test pattern (Humphrey Visual Field Analyser II; Carl Zeiss Meditec, Dublin, CA), and pattern standard deviation (PSD) and Mean Deviation (MD) were noted.

Measurement of the subfoveal choroidal thickness was done using SD-OCT: Optovue RTVue 100 (Optovue Inc., Fremont, CA, USA). The choroidal thickness was measured manually at the subfovea starting from the outer portion of the hyperreflective line corresponding to the RPE to the inner surface of the sclera, using the line segment measurement tool (Fig. 1). The choroid was measured by two independent graders who were blinded to the diagnosis, then their measurements were averaged.

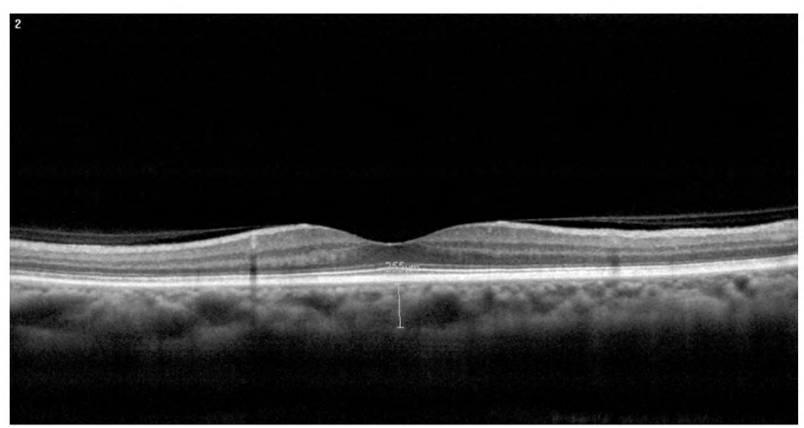

Fig. (1): Subfoveal choroidal thickness of one of our patients $=255$ r c

\section{Results}

The study included 124 eyes.

\section{Demographic data:}

Fig. (2) shows gender distribution in each group.

Table (1) and Fig. (3) show mean of age in each group.

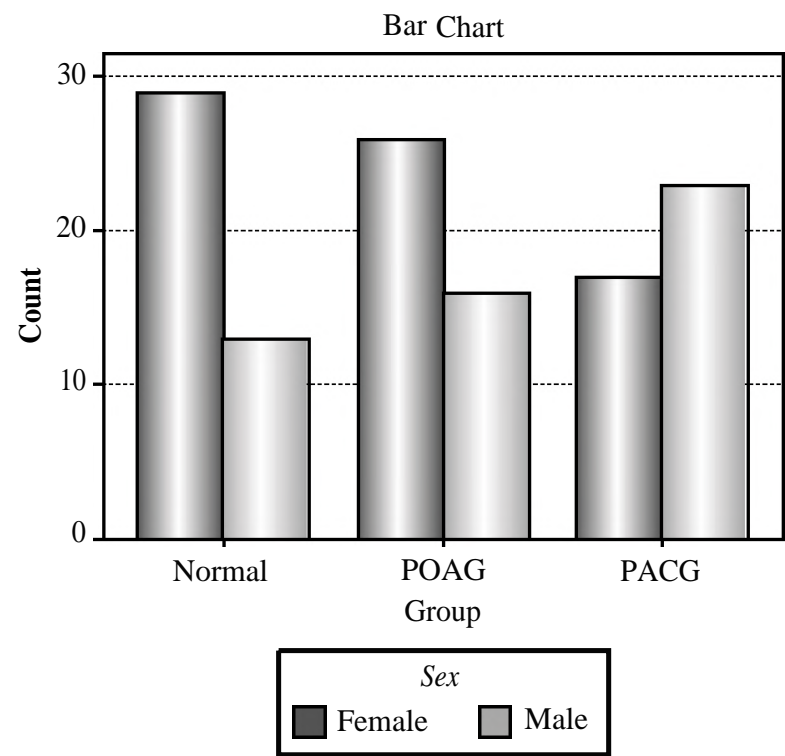

Fig. (2): Gender distribution within the three groups.

Table (1): Age.

Normal POAG PACG Total Ralue

Mean of age $56.4 \pm 7.553 .8 \pm 6.854 .4 \pm 7.755 .5 \pm 7.60 .09$

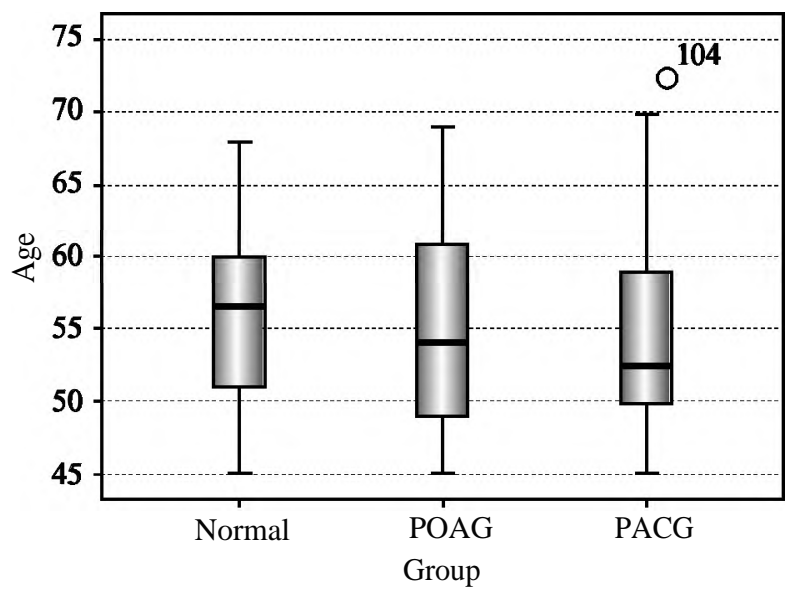

Fig. (3). Age within the three groups.

\section{Regarding the subfoveal choroidal thickness:}

The normal group had thicker subfoveal choroid than both POAG and PACG groups, but their relationship was not statistically significant (post-Hoc, $p$-values $=0.7 \& 1.0$ respectively), while $\mathrm{PACG}$ group had thicker subfoveal choroid than POAG group, but their relationship was not statistically significant either (post-Hoc, $p$-value $=0.97$ ).

The mean of average subfoveal choroidal thickness

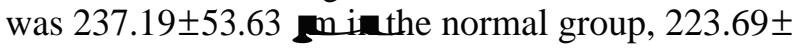
$50.81 \mathrm{~min}$ the POAG group and $235.04 \pm 51.14 \mathrm{~m}$ in the PACG group (ANOVA, $p$-value $=0.4$ ), as shown in Table (2) and Fig. (4). 
Table (2): Average subfoveal choroidal thickness

\begin{tabular}{lccc}
\hline & Normal POAG PACG & Palue \\
\hline $\begin{array}{l}\text { Mean of average } \\
\text { choroidal thickness }\end{array}$ & 53.63 & 50.81 & 51.14 \\
\hline
\end{tabular}

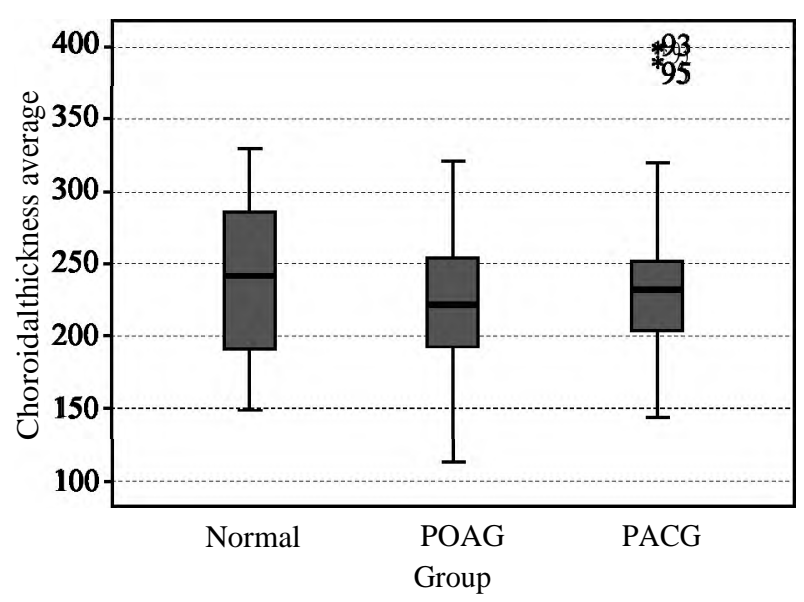

Fig. (4): Average subfoveal choroidal thickness.

Regarding the average RNFL thickness:

The normal group had significantly thicker RNFL than both POAG and PACG groups (post-Hoc, both $p$-values $<0.001$ ), while the POAG group had thicker RNFL than PACG group, but their relationship was not statistically significant (post-Hoc, $p$-value $=1.0$ ).

The mean of average RNFL thickness was 110.02 $\pm 10.3 \mathrm{~min}$ the normal group, $94.44 \pm 21.63 \mathrm{~min}$ POAG group and $91.01 \pm 20.89 \mathrm{~m}$ inthe PACG group (ANOVA, $p$-value $<0.001$ ), as shown in Table (3) and Fig. (5).

Table (3): Average RNFL thickness.

\begin{tabular}{cccc}
\hline & Normal POAG PACG & $\begin{array}{c}p- \\
\text { value }\end{array}$ \\
\hline Mean of average & $110.02 \pm 94.44 \pm 9$ & $1.01 \pm<0.001$ \\
RNFL thickness & $\mathbf{1 0 . 3}$ & 21.63 & 20.89 \\
\hline
\end{tabular}

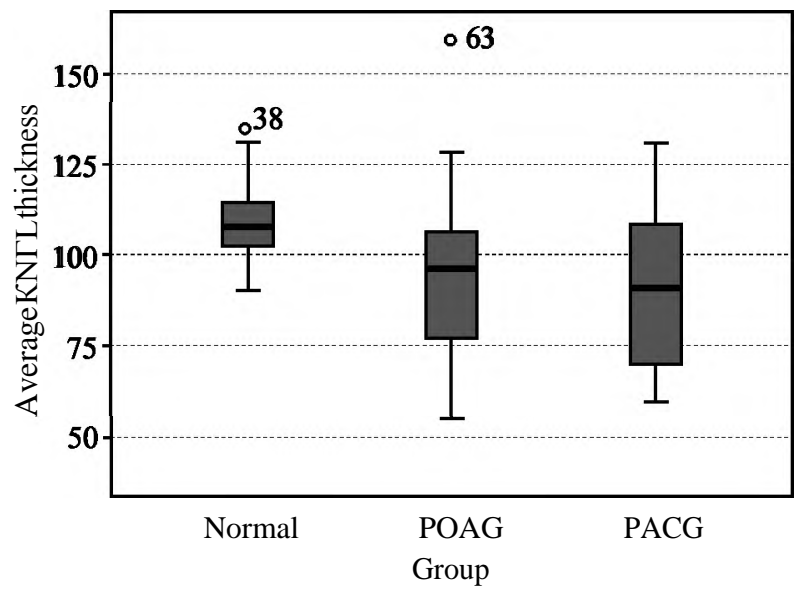

Fig. (5): Average RNFL thickness.
Regarding the average GCC thickness:

The normal group had significantly thicker GCC than both POAG and PACG groups (post-Hoc, $p$ values $=0.006 \&<0.001$ respectively), while the POAG group had thicker GCC than PACG group, but their relationship was not statistically significant (post-Hoc, $p$-value $=0.16$ ).

The mean of average GCC thickness was 96.53 \pm 6.59 in the normal group, $88.36 \pm 13.5$ in the POAG group and $83.32 \pm 13.82 \mathrm{~m}$ in the PACG group (ANOVA, $p$-value $<0.001$ ) as shown in Table (4) and Fig. (6).

Table (4): Average GCC thickness.

\begin{tabular}{clll}
\hline & \multicolumn{2}{l}{ Normal POAG PACG } & $\begin{array}{c}p- \\
\text { value }\end{array}$ \\
\hline Mean of average & $96.53 \pm$ & $88.36 \pm$ & $83.32 \pm<0.001$ \\
GCC thickness & 6.59 & 13.5 & 13.82 \\
\hline
\end{tabular}

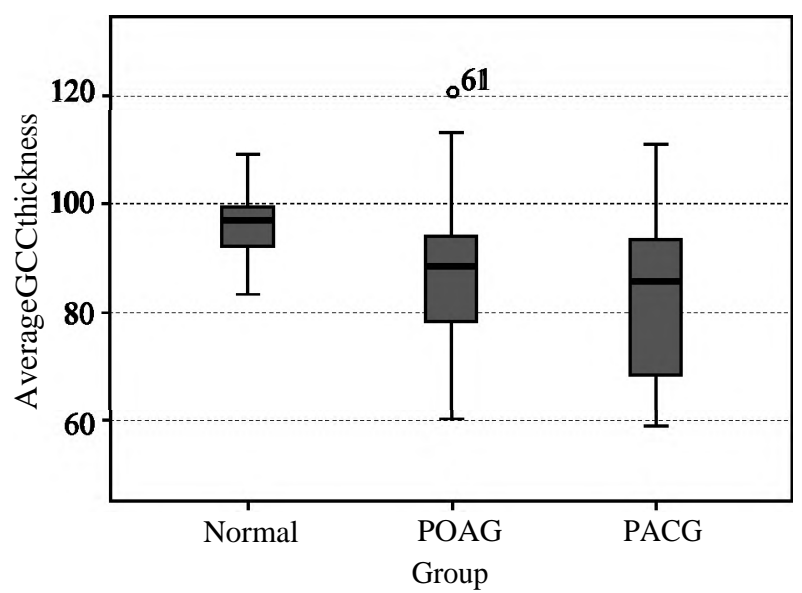

Fig. (6): Average GCC thickness.

Figs. (7-9) show examples of our cases.

Correlation between subfoveal choroidal thickness and different variables:

Our study demonstrated a positive correlation between subfoveal choroidal thickness and each of:

- Average RNFL thickness ( $r=0.188, p$-value $=$ 0.036).

- Average GCC thickness $(r=0.066, p$-value $=$ $0.464)$.

- MD $(r=0.136, p$-value $=0.132)$.

While there was a negative correlation between subfoveal choroidal thickness and each of:

- IOP level $(r=-0.068, p$-value=0.455).

- PSD $(r=-0.122, p$-value $=0.177)$. 

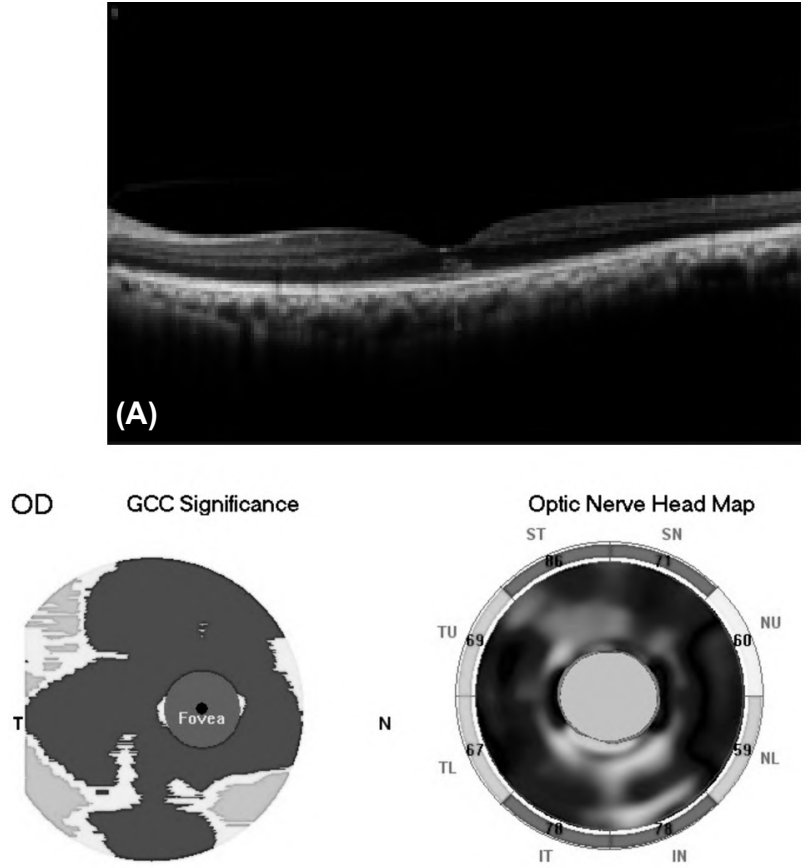

Exam Date: $09 / 17 / 2014$, SSI $=71$

N
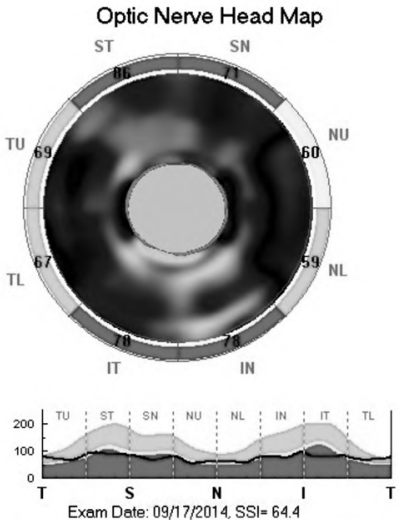

(C)

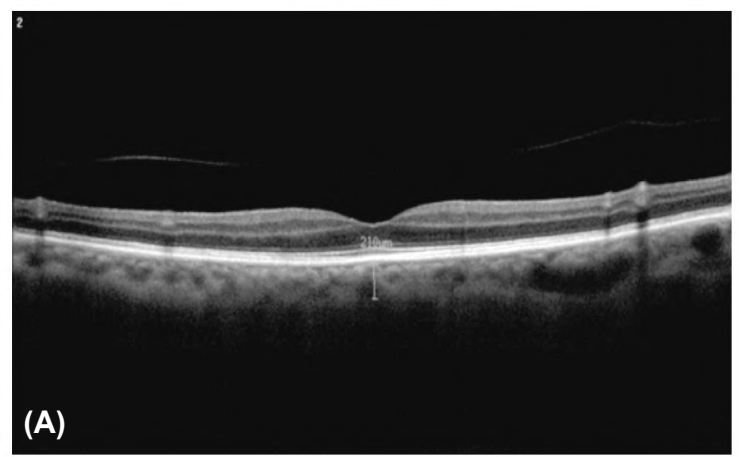

OD

GCC Significance

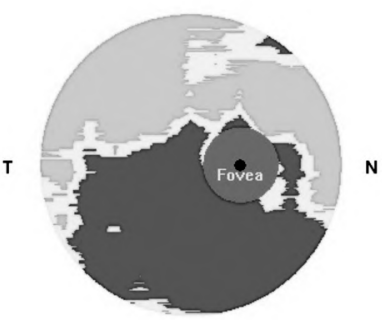

(C)

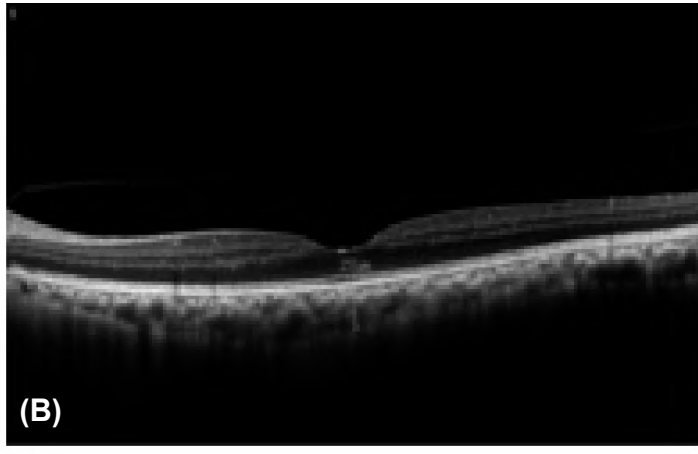

\begin{tabular}{|c|c|c|}
\hline RNFL Parameters & & os \\
\hline Avg. RNFL & 7.08 & 0.00 \\
\hline Sup. Avg & 1.52 & 0.00 \\
\hline Inf. Avg & 0.64 & 0.00 \\
\hline Nerve Head Parameter & ers $O D$ & os $>>$ \\
\hline Rim Yolume (mm³) & 0.001 & 10.000 \\
\hline Nerve Head Vim (mm²) & ) 0.004 & 40.000 \\
\hline Cup Volume (mm³) & 1.280 & 0.000 \\
\hline Nerve Head Parsmeter & ers $O D$ & os \\
\hline Optic Disk Area (mm²) & 2.47 & 0.00 \\
\hline CupiDisc Area Ratio & 0.91 & 0.00 \\
\hline Horizontal Cio Ratio & 1.00 & 0.00 \\
\hline Vertical CIID Ratio & 1.00 & 0.00 \\
\hline Rim Area $\left(\mathrm{mm}^{2}\right)$ & 0.23 & 0.00 \\
\hline Cup Area $\left(\mathrm{mm}^{2}\right)$ & 2.25 & 0.00 \\
\hline $\begin{array}{l}\text { p>5\% Within Norm } \\
\text { p<5\% Borderline } \\
p<5 \% \text { curgle Ner }\end{array}$ & & \\
\hline GCC Parameters & $O D$ & os \\
\hline Avg. $\operatorname{GcC}(\mu \mathrm{m})$ & 61.64 & 0.00 \\
\hline Sup. $G \subset C(\mu \mathrm{m})$ & 8.75 & 0.00 \\
\hline Inf. $G \subset c(\mu \mathrm{m})$ & 1.59 & 0.00 \\
\hline FLV (\%) & 1969 & 0.000 \\
\hline $\operatorname{GL}(\%)$ & 13361 & 0.000 \\
\hline
\end{tabular}

Fig. (7): OCT of a PACG eye in our study. (A) Subfoveal choroidal thickness measured by the first observer $=25111$, (B) Subfoveal choroidal thickness measured by the second observer=21111, (S) RNFL \& GCC thickness.

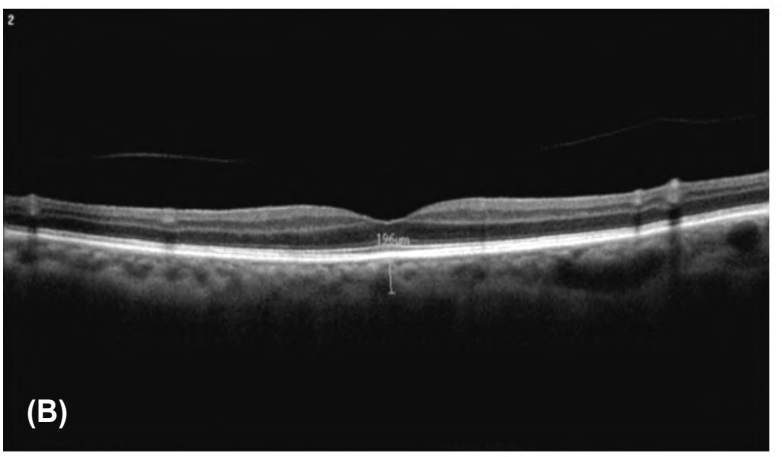

\begin{tabular}{|c|c|c|}
\hline NFL Parameters & $O D$ & 05 \\
\hline Ayg. RNFL & 73.81 & 90.95 \\
\hline Sup. Avg & 7.00 & |91.03 \\
\hline Inf. Avg & 70.62 & 90.86 \\
\hline Nerve Head Parmete & ters $\mathrm{OD}$ & os \\
\hline Rim Volume (mm²) & 0.032 & 20.021 \\
\hline Nerve Head vllm (m.32) & 2) 0.068 & 80.047 \\
\hline Cup Volume (mm²) & 0.693 & $\begin{array}{ll}30.396 \\
\end{array}$ \\
\hline Nerve Head Paramete & ters $O D$ & os \\
\hline Optic Disk Area $\left(\mathrm{mm}^{2}\right)$ & 2) 2.04 & 2.00 \\
\hline CupjDisc Area Ratio & 0.71 & 0.74 \\
\hline Horizontal C/D Ratio & 0.94 & 0.99 \\
\hline Nertical C/D Ratio & 0.94 & 0.92 \\
\hline Rim Area (mm²) & 0.59 & 0.52 \\
\hline Cup Area $\left(\mathrm{mm}^{2}\right)$ & 1.45 & 1.48 \\
\hline 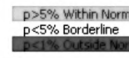 & & \\
\hline GCC Parameters & $O D$ & os \\
\hline Avg. $G \subset C(\mu m)$ & 72.08 & 85.96 \\
\hline Sup. $G C C(\mu m)$ & 80.83 & 89.13 \\
\hline Inf. $\operatorname{GcC}(\mu \mathrm{m})$ & 63.34 & 82.81 \\
\hline FLY (\%) & 9.578 & 0.848 \\
\hline $\operatorname{GLV}(\%)$ & 4.269 & 10.446 \\
\hline
\end{tabular}

Fig. (8): OCT of a POAG eye in our study. (A) Subfoveal choroidal thickness measured by the first observer=210 (B) Subfoveal choroidal thickness measured by the second observer=196 (C) RNFL \& GCC thickness. 

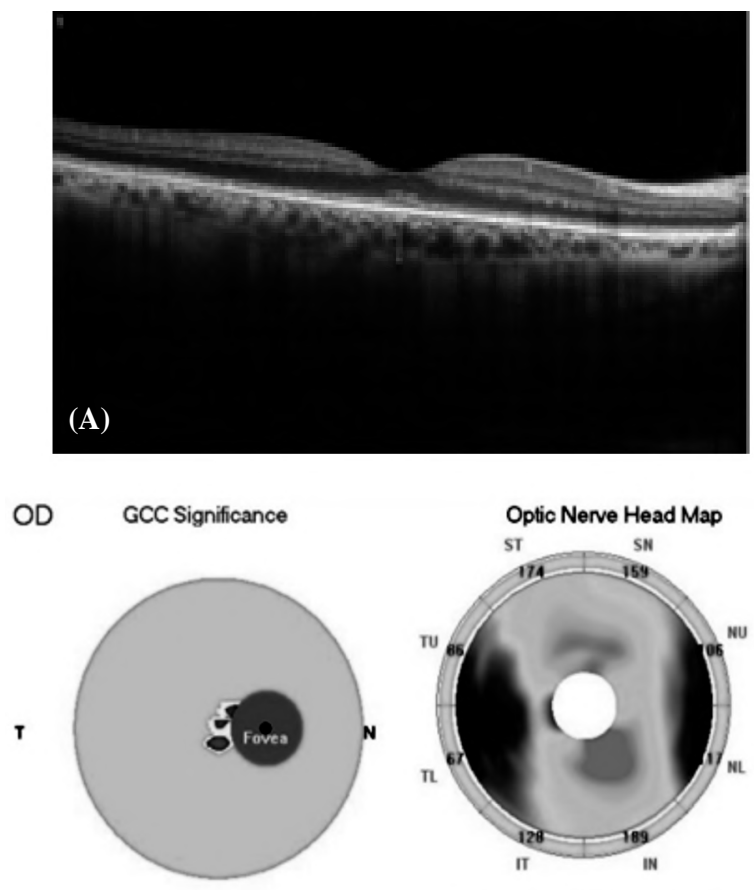

Exam Date. 041772016. SS1 59.6

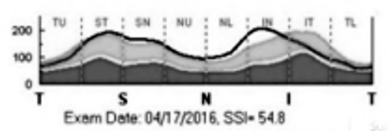

(C)
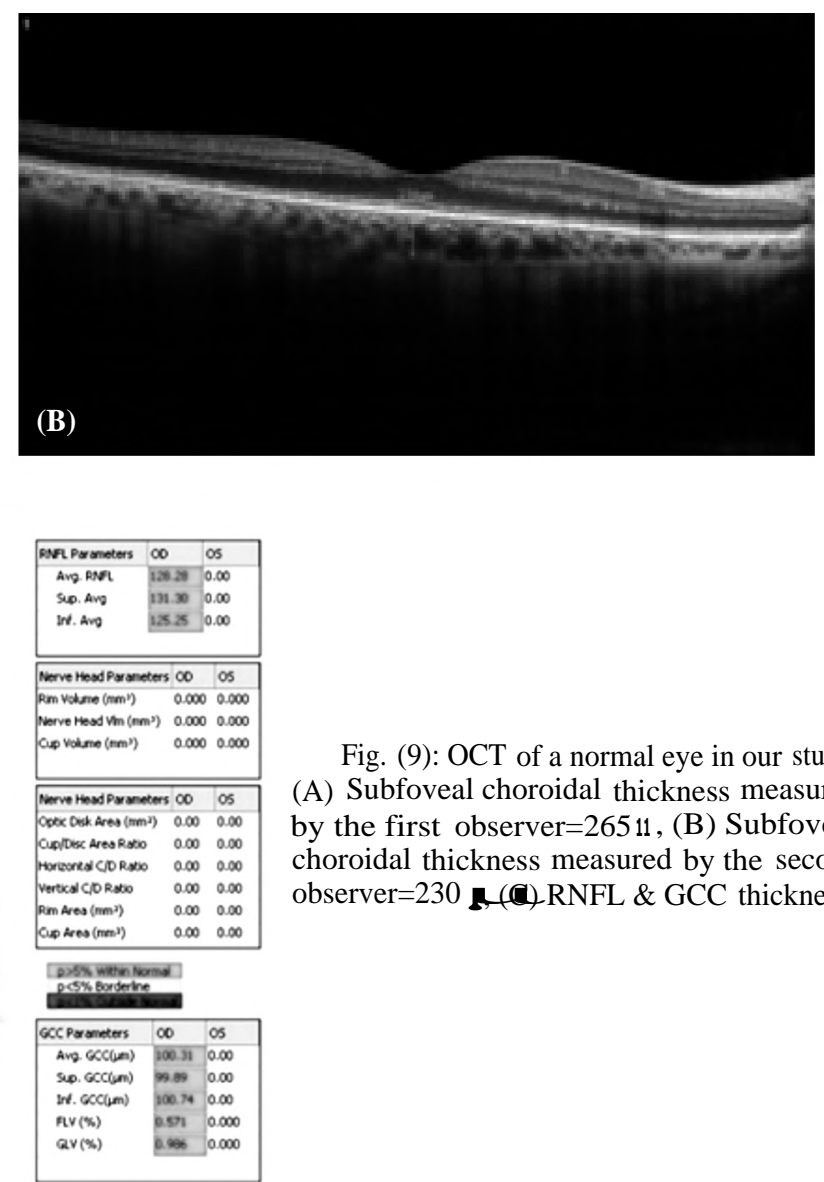

Fig. (9): OCT of a normal eye in our study. (A) Subfoveal choroidal thickness measured by the first observer $=26511$, (B) Subfoveal choroidal thickness measured by the second observer=230 RNFL \& GCC thickness.

\section{Discussion}

The main finding of our study is the lack of a significant difference in subfoveal choroidal thickness among normal eyes, eyes with POAG and eyes with PACG, where the normal group had thicker subfoveal choroid than both POAG and PACG groups, but their relationship was not statistically significant ( $p$-values $=0.7 \& 1.0$ respectively), while PACG group had thicker subfoveal choroid than POAG group, and their relationship was not statistically significant either $(p$-value $=$ 0.97 ). This finding may reflect a lack of significant association between glaucoma and subfoveal choroidal thickness.

Mwanza et al., [6] reported their experience with in vivo imaging of the choroid with Spectralis SD-OCT in primary open-angle and normal-tension glaucoma and normal control subjects. They confirmed that choroidal thickness was mainly influenced by age and axial length (thinner choroid with older age and longer axial length). However, the authors did not find any significant difference among the three groups with respect to choroidal thickness under the foveal center or up to $3 \mathrm{~mm}$

nasal or temporal to the fovea. In another study by Mwanza et al. on patients with unilateral advanced glaucoma the same findings were confirmed [11], suggesting a lack of association not only between choroidal thickness and glaucoma but also with glaucoma severity.

Hosseini and colleagues [12] presented a study on macular and peripapillary choroidal thickness in patients with perimetric glaucoma. They did not find any significant difference in subfoveal choroidal thickness between patients with open angle glaucoma and non-glaucomatous individuals. They extended the examinations into the peripapillary region in which, except for the temporal region, choroidal thickness did not differ between glaucomatous and control eyes. These findings may imply that choroidal blood circulation may not be markedly involved or affected in open angle glaucoma. However, the choroid is a highly dynamic vascular tissue and purely anatomic measurements, such as choroidal thickness, may not adequately describe altered hemodynamic physiology in various ocular diseases. Specifically, flow patterns in the peripapillary choroid would be of great interest in glaucoma [12]. 
The findings by the above-mentioned studies are consistent with our results regarding the lack of a significant difference between the subfoveal choroidal thickness in POAG and normal eyes.

Contrary to this, Bron et al., [7] reported thinner subfoveal choroidal thickness in POAG patients than normal controls in a study which involved 65 healthy eyes and 78 eyes with POAG. Also, a metaanalysis conducted by Lin et al., [13] demonstrated that average peripapillary choroidal thickness in open-angle glaucoma was significantly reduced compared to healthy individuals which was a potential support of the vascular theory of glaucoma and suggested that retrobulbar ischemia might have an impact on the optic nerve head. The difference between these results and ours may be attributed to our relatively smaller sample size.

With respect to choroidal thickness in angle closure glaucoma, Arora and colleagues [14] reported that choroidal thickness was significantly greater in the angle closure glaucoma group than in the open angle glaucoma group and normal subjects, with no significant difference between eyes with open angle glaucoma and normal subjects.

In another study on fellow eyes of 44 patients with unilateral acute primary angle closure, Zhou and colleagues [15] observed that the unaffected fellow eyes had thicker choroid than a group of control eyes.

The difference in results between our study and the above-mentioned studies regarding choroidal thickness in PACG may be attributed to the relatively smaller sample size in our study. This is in contrast to the study by Arora et al., [14], which involved 40 controls, 106 POAG and 79 PACG eyes.

In addition, we observed a positive correlation between average RNFL thickness and average GCC thickness $(r=0.804, p$-value $<0.001)$, a positive correlation between average RNFL thickness and MD ( $r=0.690, p$-value <0.001), and a negative correlation between average RNFL thickness and PSD ( $r=-0.461, p$-value $<0.001)$. All of these relations were statistically significant.

We also found a positive correlation between choroidal thickness and the average RNFL thickness $(r=0.188, p$-value $=0.036)$ which was statistically significant, while the same positive relation was found with the average GCC thickness ( $r=$ $0.066, p$-value $=0.464)$ and MD ( $r=0.136, p$-value $=0.132$ ) but it was not statistically significant. These findings reflect a negative correlation be- tween choroidal thickness and glaucoma severity as defined by structural and functional measures (RNFL thickness \& GCC thickness, and MD respectively).

In addition, in the present study there was a negative correlation between subfoveal choroidal thickness and PSD ( $r=-0.122, p$-value $=0.177$ ) which also implies a negative correlation between choroidal thickness and glaucoma severity, but their relationship was not statistically significant.

This is in contrast to Arora et al., [14] who found that the severity of glaucomatous optic nerve damage as measured by cup/disc ratio or visual field mean deviation was not significantly associated with choroidal thickness. Similarly, Hosseini et al., [12] observed that glaucoma severity (in terms of RNFL thickness and MD) was not associated with choroidal thickness. These findings are consistent with those of the previously-mentioned study by Mwanza et al., [11], involving patients with unilateral advanced glaucoma. Likewise, Bron, et al., [7] found that subfoveal choroidal thickness was not different according to glaucoma stage.

\section{Conclusion:}

No significant difference in subfoveal choroidal thickness among normal eyes, eyes with POAG and eyes with PACG. Subfoveal choroidal thickness had significant positive correlation with average RNFL thickness.

\section{References}

1- QUIGLEY H.A. : Neuronal death in glaucoma. Prog. Retin. Eye. Res., 18: 39-57, 1999.

2- HEIJL A., LESKE M.C., BENGTSSON B., et al.: Reduction of intraocular pressure and glaucoma progression: results from the Early Manifest Glaucoma Trial. Arch. Ophthalmol., 120: 1268-1279, 2002.

3- SUGIYAMA T., SCHWARTZ B., TAKAMOTO T., et al.: Evaluation of the circulation in the retina, peripapillary choroid and opticdisk in normal-tension glaucoma. Ophthalmic Res., 32: 79-86, 2000.

4- FUCHSJAGER-MAYRL G., WALLY B., GEORGOPOULOS M., et al.: Ocular blood flow and systemic blood pressure in patients with primary open-angle glaucoma and ocular hypertension. Invest. Ophthalmol. Vis. Sci., 45: 834-839, 2004.

5- GALASSI F., SODI A., UCCI F., et al.: Ocular hemodynamics and glaucoma prognosis: A color Doppler imaging study. Arch. Ophthalmol., 121: 1711-1715, 2003.

6- MWANZA J-C., HOCHBERG J.T., BANITT M.R. et al.: Lack of Association between Glaucoma and Macular Choroidal Thickness Measured with Enhanced Depth- 
Imaging Optical Coherence Tomography. Invest. Ophthalmol. Vis. Sci., 52: 3430-3435, 2011.

7- BRON A., FRANCOZ A., BEYNAT J., et al.: Is choroidal thickness different between glaucoma patients and healthy subjects?. Acta Ophthalmologica, 89: 1755-1768. doi: 10.1111/j.1755-3768.2011.4353, 2011.

8- HIROOKA K., TENKUMO K., FUJIWARA A., et al.: Evaluation of peripapillary choroidal thickness in patients with normal-tension glaucoma. BMC Ophthalmology, 12: 29 doi:10.1186/1471-2415-12-29, 2012.

9- SPAIDE R.F., KOIZUMI H., POZZONI M.C.: Enhanced depth imaging spectral-domain optical coherence tomography. Am. J. Ophthalmol., 146 (4): 496-500, 2008.

10- BRANCHINI L., REGATIERI C.V., FLORES-MORENO I., et al.: Reproducibility of Choroidal Thickness Measurements acrossThree Spectral Domain Optical Coherence Tomography Systems. Ophthalmology, 119 (1): 119-123, 2012.
11- MWANZA J., SAYYAD F. and BUDENZ D.: Choroidal thickness in unilateral advanced glaucoma. Invest. Ophthalmol. Vis. Sci., 53 (10): 6695-6701, 2012.

12- HOSSEINI H., NILFORUSHAN N., MOGHIMI S., et al.: Peripapillary and macular choroidal thickness in glaucoma. J. Ophthalmic. Vis. Res., 9: 154-161, 2014.

13-LIN Z., HUANG S., XIE B., et al.: Peripapillary Choroidal Thickness and Open-Angle Glaucoma: A Meta-Analysis. Journal of Ophthalmology, 2016, Article ID 5484568: 112, 2016.

14- ARORA K.S., JEFFERYS J.L., MAUL E.A., et al.: The choroid is thicker in angle closure than in open angle and control eyes. Invest. Ophthalmol. Vis. Sci., 53: 78137818, 2012.

15-ZHOU M, WANG W, DING X., et al.: Choroidal thickness in fellow eyes of patients with acute primary angle-closure measured by enhanced depth imaging spectral-domain optical coherence tomography. Invest. Ophthalmol. Vis. Sci., 54: 1971-1978, 2013.

\section{دراسة مقارنة بين سمك المشيمة تحت ماقولة العين

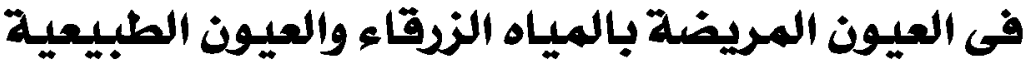

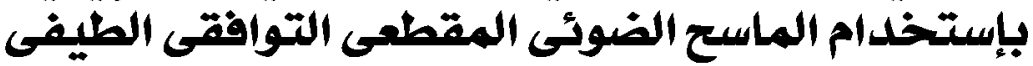

لطالما كان دود مسيمة العين مثيراً للأنتباه فى حدوث الاعتلال العصبى البصرى الزرقى. كان الهدف من الدراسة التى قمثا بها هو مقارنة

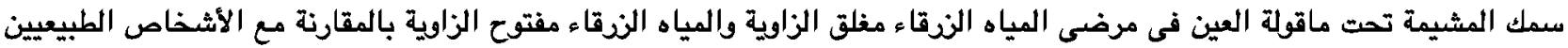

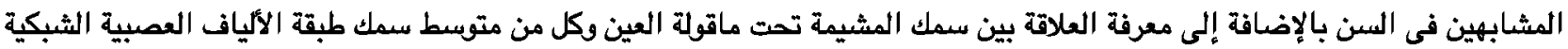

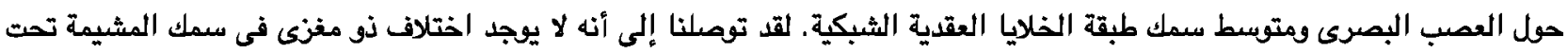

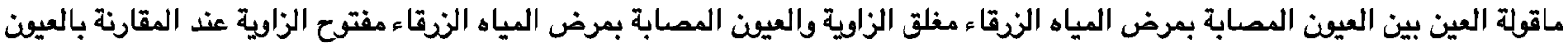

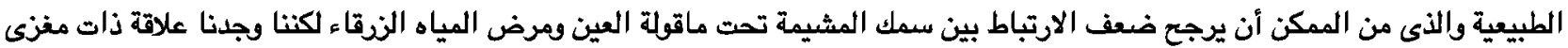

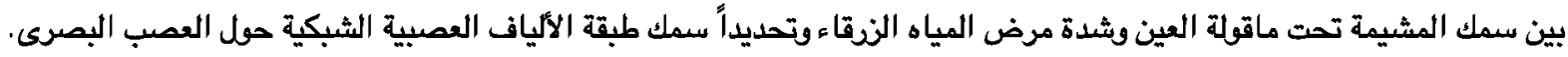

